\section{An Optical-Acoustic Method of Gas Analysis}

Dr. M. Vengerov has described a method of gas analysis under this title ${ }^{1}$. As the method has been in practical use in the chemical industry for about ten years, I should like to direct attention to the selfrecording infra-red absorption apparatus, called 'Uras', developed by Dr. Luft-Oppau.

The principle of this analyser is the same as that of Dr. Vengerov, but the arrangement is somewhat better. The radiations of two infra-red sources pass intơ two gilded glass tubes, after being interrupted periodically by a revolving disk with two sectors, and are absorbed finally in a condenser chamber separated into two parts by a thin metal foil. One glass tube contains a standard gas, and through the other streams the gas to be examined. The condenser chamber contains some of the gas under examination. Both the tubes and the condenser chamber are sealed with rock-salt windows, allowing the infra-red rays to pass through. In front of the separating foil in the condenser chamber is an insulated wire gauze arranged to form a kind of condenser microphone.

If the streaming gas contains an unknown component, this will absorb its characteristic radiation out of the infra-red beam. As the condenser chamber contains the same gas, it also will absorb only this characteristic radiation. The effect will be that the part of the condenser chamber beneath the analyser tube is not so much heated as the standard part, and the condenser foil is deflected less in relation to the wire gauze. The condenser microphone is in the grid circuit of a three-stage amplifier tuned to the frequency of the revolving disk.

The arrangement of two separated beams eliminates any effect of changes in the infra-red source, and the filling of the condenser chamber with a special gas guarantees great selective efficiency of the apparatus. If necessary, tubes containing the disturbing gas components can be placed in front of the analyser tubes. These tubes then act as spectral filters and absorb the characteristic radiations of the extraneous gases out of the two beams. By this device the presence of one of these disturbing gases in the gas to be examined causes no effect on the condenser chamber and the condenser itself.

With the 'Uras' the percentage can be recorded of any organic or inorganic gas having absorption bands within the range of the infra-red source, to which the condenser chamber has been adjusted. The sensitiveness is sufficient to record traces of the gas of the order of magnitude of fractions of a thousandth part.

178 Elbchaussee, Hamburg.

${ }^{1}$ Nature, 158, 28 (1946).

\section{Experimental Data and 'Sufficient' Accuracy}

Mr. H. A. HUGHES has pointed out ${ }^{1}$ that replication could cease when a required standard of accuracy is attained, and that, from a sample size of $n$, the estimate, $\alpha$, of the probable error of its mean “. . . decreases with ascending values of $n$ until the minimum value permitted by the experimental conditions is reached".

Since $\propto \sqrt{n}$ in general converges in the statistical sense to a finite limit-the probable error of a single observation-clearly $\alpha$ similarly converges to zero. Expressed practically, we may determine a quantit y as accurately as we please by adequate replication. provided our measurements are unbiased. The elimination of bias is not, normally, possible. While, therefore, we may reduce $\alpha$ indefinitely, the labour of further replication is unwarranted once the magnitude of $\alpha$ has been reduced to the same order as that of the bias.

A second point : as Mr. Hughes referred to measurements on several systems, I infer he may ultimately have intended to compare them. If this were his sole object in attaining a prescribed accuracy, it would be most economic in replication to apply a sequential test ${ }^{2}$. When sequential tests are impracticable, as in some agricultural and building research when each experiment takes many months, it is possible to plan a priori to attain a required certainty of avoiding inadequate replication. It is intended to publish a paper on this in the near future, though it is suspected that reports, probably in the agricul. tural field, must already exist of prior research on this topic.

Planning and Analysis Section,

Chief Scientific Advisor's Division, Ministry of Works.

Dec. 2.

Nature, 158, 29 (1946)

2 Wald, A., Ann. Math. Stat., 18 (1945).

\section{N. H. CARrier}

Wald, A., Ann. Math, Stat, 18 (1045).

\section{Fluid Flow at a Small Hole due to Vibration}

I sHouLD like to acknowledge my indebtedness to Prof. Andrade ${ }^{1}$ for directing my attention to the work of $\mathrm{H}$. Sell ${ }^{2}$. As a result, I have made a careful examination, by means of strong lateral illumination, of the way in which the smoke particles enter and leave the orifice in the resonator. There is no doubt about the general accuracy of H. Sell's explanation. and this is particularly well demonstrated when the smoke is made to issue in the space between two parallel plates of glass kept apart by a distance piece the thickness of which is approximately that of the diameter of the orifice. The smoke particles can then be seen as bright dots on a dark ground, and their paths easily traced. As well as the back-flow near the surface of the resonator, vortex motion occurs. If, however, the smoke be allowed to issue from a $V$-shaped mouthpiece formed by sealing two flat pieces of glass between the plates, then, provided the excitation is not too powerful, both the vortex motion and the back-flow cease. There now occurs what appears to the eye as a slow steady motion of the smoke outwards. Examination of the particles with a low-power microscope shows them, however. no longer as dots but as little lines, so that a vibratory motion is superimposed on the general forward motion.

It would appear, therefore, at first sight, that the experiment is now on a more satisfactory basis. When the resonator is constructed of glass, however. vortex motion inside the tube in the neighbourhood of the orifice can be seen. Particles within the tube travel normally away from the centre of the orifice, pursue a more or less circular path and return along the base to the orifice once more. Some of these particles, however, move outwards through the orifice, and these constitute the slow steady stream 